Pecvnia, Monográfico (2008), pp. 1-18

\title{
Cuando su fabricante contratista se convierte en su competidor
}

\author{
Benito Arruñada \\ benito.arrunada@upf.edu \\ Universidad Pompeu Fabra \\ Dpto. de Economía y Empresa \\ Ramon Trias Fargas, 25 \\ 08005 Barcelona (España) \\ Xosé H. Vázquez \\ xhvv@uvigo.es \\ Universidad de Vigo \\ Dpto. de Organización de Empresas y Marketing \\ Fac. de Ciencias Económicas y Empresariales \\ Rúa Leonardo da Vinci \\ Campus das Lagoas / Marcosende \\ 36310 Vigo (España)
}

La fabricación ha sido el aspecto fundamental de las empresas manufactureras durante más de un siglo. Sin embargo, en la actualidad, cada vez más compañías están subcontratando su
Manufacturing has been the core of industrial firms for more than a century. Nowadays, however, an increasing number of companies are contracting out their production

Traducido y reimpreso con permiso de Harvard Business Review. Este artículo fue publicado originalmente bajo el título inglés de "When your contract manufacturer becomes your competitor" de Benito Arruñada y Xosé H. Vázquez en el número de Septiembre de 2006. Copyright $\odot$ (2006) by the Harvard Business School. Publishing Corporation; all rights reserved. This translation, Copyright $\odot$ (2007) by the Harvard Business School Publishing Corporation. 
producción a empresas especializadas. En algunas de ellas, los trabajadores no llegan a establecer un contacto físico con los productos. El cambio tecnológico no sólo está promoviendo la especialización mediante la utilización de robots y fábricas flexibles, sino que además está reduciendo los costes de coordinación a través de la aplicación de nuevos estándares y el uso de Internet. Este es el motivo por el que la subcontratación de la fabricación ("contract manufacturing", en inglés) puede predominar en un futuro próximo. Desafortunadamente, los incentivos de los directivos fomentan una aproximación general y carente de análisis a las decisiones de externalizar o no, qué productos externalizar, con qué subcontratistas fabricantes (CM, por "Contract Manufacturer", en inglés) comprometerse, y de qué forma -un acuerdo de mercado, una alianza estratégica, o alguna forma intermedia. Cuando los fabricantes de equipos originales (OEM, por "Original Equipment Manufacturer", en inglés) comparten aspectos de propiedad intelectual básicos con sus CMs, es importante que la relación sea cercana y de confianza -pero no tan estrecha como para que los CMs se alejen del mercado y de las contribuciones de otros OEMs. with specialists. In some of them not even a single worker touches physically the products it sells. Technological change is not only fostering specialization through the use of robots and flexible factories, but also reducing coordination costs by applying new standards and using the Internet. This is the reason why this "contract manufacturing" might well become the rule in the near future. Unfortunately, managers' incentives encourage a wholesale, uncritical approach to decisions about whether to outsource, which products to outsource, which CMs to engage, and in what form-a market agreement, a strategic alliance, or something in between. When OEMs share sensitive intellectual property with CMs, it is important that the relationship be trusting and close-but not so close that CMs lose touch with the market and other OEMs' contributions.

\section{Numerosos subcontratistas contratados (Contract}

Manufacturer, CM) por los fabricantes de equipos originales (Original Equipment Manufacturer, OEM), gracias al desarrollo de nuevas fórmulas contractuales, han permitido a estos últimos externalizar las actividades de fabricación, reducir costes y centrarse en la investigación, el diseño y la comercialización de nuevos productos. Algunos subcontratistas contratados (CM), los más hambrientos, con estrategias agresivas, están empezando a morder la mano que los alimenta. Los fabricantes de equipos originales (OEM) más hábiles han conseguido mantener estos riesgos bajo control.

IBM creó la industria de los ordenadores portátiles. Sin embargo, no pasará mucho tiempo antes de que el logotipo de la empresa desaparezca de los ordenadores e IBM abandone el negocio, excepto por la joint venture que ha formado recientemente con el fabricante de PC, Lenovo. Creado en 1984 como distribuidor en China de equipos fabricados por IBM y otras empresas, Lenovo pondrá finalmente su propio logotipo en los PC. Ciertamente, la empresa ha avanzado un largo camino. También 
lo ha hecho Sanmina-SCI, la cual es fabricante de algunos PC de IBM en Estados Unidos y que recientemente ha adquirido algunas de las fábricas donde se fabrican los ordenadores. Al igual que Lenovo, Sanmina ensambla productos para una gran variedad de marcas conocidas. Pero la firma ha ampliado su rol, y ahora también diseña y desarrolla componentes electrónicos a pedido. Estas dos empresas son representativas de una multitud de fabricantes de productos de marca, hasta hace poco anónimos, que están irrumpiendo y desplazando a las marcas mismas. De hecho, las complejidades del entorno de IBM desafían la visión común de la fabricación por contrato como nada más y nada menos que el recurso desesperado de las grandes marcas enfrentadas a márgenes de utilidad decrecientes.

Sin duda, subcontratar la fabricación completa de un producto permite a los fabricantes de equipos originales (OEM) reducir sus costes de mano de obra, liberar capital y mejorar la productividad de sus empleados. Los OEM pueden entonces concentrarse en aquellas tareas que potencian en mayor medida el valor de un producto: I\&D, diseño y marketing, por ejemplo. Para facilitar la obtención de estos beneficios están las fortalezas especiales del fabricante contratista (CM), dentro de las cuales se incluyen su ubicación en países de bajos salarios, economías de escala, destrezas de fabricación y exposición a los procesos de ingeniería y desarrollo de los productos que maneja para otros OEM (esta exposición permite al CM proponer mejoras a los productos de diferentes clientes).

Sin embargo, como IBM y otras empresas han aprendido, la fabricación por contrato es un arma de doble filo. Por un lado, un CM tiene acceso a la propiedad intelectual de un OEM, la cual podría filtrar hacia otros clientes o arrogarse para sí. Por el otro, un CM ambicioso podría reclamar para sí las ventajas mismas que provee a un OEM. Habiendo fabricado el producto de un OEM en su totalidad, el CM podría decidir crear su propia marca y forjar relaciones propias con minoristas y distribuidores, incluyendo los del OEM. Cuando estas cosas ocurren, el OEM podría verse enfrentado no sólo a competidores más peligrosos, sino también a un nuevo tipo de competidor: el en su momento subestimado $\mathrm{CM}$. $\mathrm{Y}$, lo que es peor, si el OEM no hubiera entregado su negocio al fabricante contratista, los ingresos y conocimientos de este último tal vez habrían sido insuficientes para permitirle introducirse en el mercado de su cliente.

Aunque lanzar una marca no es algo trivial para ningún fabricante contratista, una identidad de marca arraigada en las destrezas 
productivas del CM tendría credibilidad inmediata. Además, un CM que trabaja para varios OEM tiene experiencia fabricando una gama más amplia de productos que la mayoría de sus clientes, lo que le permite concentrarse en producir los más rentables. Y su estructura de costes no necesariamente soporta la carga de inversiones en I\&D.

En resumen, los humildes intentos de los OEM por obtener mejoras operacionales y ahorros de costes pueden arrastrarlos hacia un terrero estratégicamente traicionero, en el cual los socios rápidamente se superan los unos a los otros, espían oportunidades más atractivas en otros lugares y, en los casos más flagrantes, muerden la mano que los ha estado alimentando. Dicho de forma simple, los OEM que emplean a fabricantes contratistas pueden liberar fuerzas que sean difíciles de controlar. No es exagerado afirmar que los actores muy pronto se hallan inmersos en un melodrama cargado de promiscuidad (CM que persiguen relaciones con una variedad de OEM), infidelidad (minoristas y distribuidores que le entregan su negocio al CM de un OEM) y traición (CM que transmiten la propiedad intelectual de un OEM a sus rivales o se la apropian).

Los OEM no pueden eludir este dilema poniendo término a sus acuerdos de subcontratación: el diseño modular, la codificación de los procesos de fabricación y los menores costes de transacción hacen irresistible la fabricación por contrato para los OEM menos capitalizados. Pero los OEM sí pueden gestionar sus relaciones con los CM de tal manera que ni se debiliten ni fortalezcan demasiado al $\mathrm{CM}$. Para hacerlo requieren algunas cosas: discreción a la hora de revelar sus secretos, cautela a la hora de elegir con quién relacionarse, y un grado cabal de intimidad, lealtad y generosidad hacia los propios socios y clientes. Los OEM también pueden evitar las tendencias traidoras de los CM usando su excedente de propiedad intelectual para introducirse en mercados distintos a los de sus productos centrales. Paradójicamente, las capacidades invasoras de los CM, que de otro modo serían usadas para introducirse en los mercados de los OEM, pueden brindar a estos últimos el acceso a nuevos mercados, y en ocasiones una salida al dilema.

\section{COMPETENCIA INTENSA}

Pocas empresas industriales todavía consideran la fabricación como una parte esencial de su negocio. Los propietarios de marcas tradicionales -a quienes hoy conocemos como OEM- prefieren centrarse 
ahora en la investigación, el diseño y la venta de productos, dejando la producción a los nuevos especialistas: los fabricantes contratistas.

La fabricación por contrato implica subcontratar completamente un proceso de fabricación hasta el punto que, en muchos casos, ninguno de los empleados de la OEM habrán tocado físicamente el producto que están comercializando y vendiendo. Esta práctica comenzó en 1981, con la fabricación de los primeros PC de IBM, aunque transcurrió una década antes de que alcanzara a productos tan cotidianos como juguetes, ropa, calzado, cerveza y productos farmacéuticos. En la actualidad, incluso algunos sectores de la industria automovilística la han adoptado: la finlandesa Valmet, Automotive ensambla el Porche Boxster, y la austriaca Magna Steyr ensambla automóviles para Mercedes, BMW y Saab.

La difusión de la fabricación por contrato ha intensificado la competencia en algunos sectores de 4 maneras:

- Creación de nuevas empresas. La fabricación por contrato facilita la creación de nuevas empresas y divisiones. Las compañías que subcontratan no tienen que reunir, invertir y arriesgar el capital necesario para desarrollar sus propias instalaciones de producción. De esa forma, pueden sortear los impedimentos tradicionales para la introducción en nuevos mercados. De hecho, cualquier empresa -incluso una que venda volúmenes bajos- puede disminuir sus costes unitarios simplemente contratando al CM de mayor escala. Así es como Dell y Gateway han sido capaces de aventurarse más allá de su raíces en los ordenadores personales e introducir a sus mercados domésticos de televisores plasma y $L C D$, reproductores de DVD y más de 50 nuevos productos.

- Creación de nuevas marcas. La situación en evolución de los fabricantes contratistas los incentiva a desarrollar sus propias marcas. Lo que ocurre es lo siguiente: a medida que los CM alcanzan una escala eficiente, sus niveles de costes convergen. Al mismo tiempo, los productos que fabrican comienzan a comoditizarse. En respuesta, los CM intentarán recuperar una ventaja competitiva sostenible emprendiendo las actividades de creación de valor que sus clientes habían realizado por cuenta propia, tales como I\&D y marketing. En una variante del dilema del innovador, los OEM ceden funciones específicas a sus CM y, al hacerlo, les dejan espacio para desarrollar las capacidades que más tarde podrían usar para amenazar a los OEM. Para entonces, los CM se habrán convertido ellos mismos en OEM. Lenovo y los fabricantes contratistas Haier (electrodomésticos) y 
TCL (televisores), las cuales también tienen su sede en China, se han transformado en tres de las mayores compañías del mundo en sus respectivos sectores precisamente de esta forma.

Si los CM descubren que no pueden obtener de sus clientes todo el conocimiento que requieren para vender y dotar de marca a un nuevo producto, pueden adquirir divisiones completas de OEM. La taiwanesa BenQ hizo precisamente eso, comprando el negocio de telefonía móvil de Siemens en 2005. Al hacerlo, BenQ adquirió no sólo la propiedad intelectual de Siemens, sino también sus décadas de experiencia de gestión, sus reservas de talento altamente desarrolladas, su marca ampliamente conocida y su plataforma global de operaciones. Una vez que los CM han alcanzado maestría en la fabricación, pueden comenzar a innovar, algo que llevan haciendo durante un tiempo: el creciente volumen de solicitudes de patentes chinas presentadas bajo el tratado europeo de patentes - 26 en 1990 y 961 en 2000- ofrece, al menos, una prueba indirecta de esto. Los CM también pueden comprar conocimiento de I\&D de los OEM. En 2004, Shanghai Automotive Industry Corporation (SAIC), la cual había realizado contratos de fabricación para Volkswagen y GM, adquirió de la quebrada MG Rover los planos necesarios para fabricar el Rover 25, el Rover 45 y el Rover 75. SAIC planea vender su propia línea de automóviles en China, Europa y Norteamérica.

- Desplazamientos por la cadena de valor. Los minoristas y distribuidores más poderosos pueden contratar a los CM más grandes y eficientes para producir (bajo las marcas propias del minorista o distribuidor) artículos de igual calidad que los de los mejores OEM. Estos productos comparten espacio en los estantes con los productos de los OEM, pero a la mitad de precio. El fabricante contratista Solectron desarrolló su pericia en fabricación trabajando para IBM, Hewlett-Packard y Mitsubishi. Luego, el distribuidor Ingram Micro pidió a Solectron que fabricara PC, servidores y otros equipos de ordenadores a pedido, bajo su propia marca y la de otros minoristas. Cadenas como Best Buy, Carrefour, Sears y Wal-Mart también están vendiendo productos electrónicos bajo sus marcas propias, diluyendo así la influencia de marketing de los OEM. Los fabricantes contratistas que han establecido sus marcas propias también sufren como consecuencia, porque a sus productos les resulta difícil mejorar la calidad, innovación y precio de los minoristas. Pero sin un CM emprendedor amenazara con dejar de fabricar artículos para un minorista, sólo empujaría a su cliente a los brazos de otro CM, uno capaz de traducir el negocio adicional en economías de escala perfeccionadas o incluso superiores. 
- Pérdida de propiedad intelectual. La fabricación por contrato pone en juego los activos intangibles particulares de los OEM. Un CM puede explotar, para beneficio de sus propias marcas, el conocimiento de adquiere al trabajar para un OEM, o el CM puede transferir (legítimamente $\mathrm{o}$ no) este conocimiento a otros clientes OEM. Estas filtraciones pueden ocurrir incluso si el CM no hace más que ensamblar componentes fabricados por otros: las tecnologías de escáner tridimensional, el diseño asistido por ordenador y la fabricación asistida por ordenador permiten a las empresas copiar, en cuestión de horas, componentes cuyo diseño pudo haber tardado años. El potencial de abuso es alto. Por ejemplo, CFM International, una joint venture entre General Electric y el fabricante francés SNECMA que produce piezas para motores aeronáuticos, ha tenido que iniciar acciones contra talleres de reparación y acondicionamiento en EE.UU. que estaban comprando piezas falsificadas. Desde luego, los OEM pueden recurrir a la justicia, al confinamiento del infractor o al lobby. Pero ninguno de estos caminos es una panacea. Los resultados de la litigación son inciertos y pueden producirse después de años de costosos procedimientos. Mientras tanto, las utilidades siguen cayendo. Persuadir a otros actores del propio sector de rechazar al CM infractor también toma tiempo, ciertamente encuentra resistencia y envuelve el riesgo de entrar en conflicto con la legislación antimonopolio. Finalmente, a medida que se propaga la globalización, las intervenciones de gobiernos individuales se vuelven menos decisivas. En cualquier caso, los OEM necesitan a los CM para seguir especializándose, agregar valor y mantenerse competitivos.

\section{UNA RELACIÓN INEVITABLE}

La fabricación por contrato es inevitable, aunque implica riesgos insoslayables. En primer lugar, los OEM que adoptan la fabricación por contrato pueden reducir sus costes directos incluso si la cantidad de unidades que venden está muy por debajo del nivel que de otra forma requerirían para alcanzar economías de escala significativas. Considere el caso de Flextronics: la planta del fabricante contratista en Guadalajara, Méjico, puede ensamblar para Royal Philips Electronics un dispositivo que conecta los televisores a Internet a costes unitarios muy bajos, porque simultáneamente produce un dispositivo similar para Sony en una línea de producción adyacente. A su vez, trabajar para muchos OEM proporciona a los $C M$ los ingresos necesarios para seguir realizando inversiones esenciales 
en la automatización de sus fábricas. Sería más difícil para IBM, HewlettPackard o el fabricante contratista Sanmina obtener economías de escala equivalentes si sus productos fueran hechos en sus propias fábricas, y si estas fábricas produjeran exclusivamente para sus propias marcas.

Cuadro 1: ¿Qué tipo de relación desea tener con su fabricante contratista?

Los acuerdos organizacionales entre fabricantes de equipos originales y fabricantes contratistas abarcan un espectro amplio desde contratos por una sola vez hasta pactos más interdependientes que podrían ser o no ser renovados. La tabla adjunta puede ayudar a las organizaciones a determinar el nivel de compromiso -y, de esa manera, el riesgo- que están dispuestas a asumir cuando se relacionan con socios de subcontratación.

\begin{tabular}{|c|l|c|}
\hline Tipo de relación & \multicolumn{1}{|c|}{ Características } & $\begin{array}{c}\text { Nivel de compromiso } \\
\text { / coste de control }\end{array}$ \\
\hline Acuerdo de mercado & Relación por una sola vez & Bajo \\
\hline Contrato renovable & Relación continua, pero no ilimitada & Moderado \\
\hline Acuerdo marco & $\begin{array}{l}\text { Acuerdo en principio para producir modelos en un } \\
\text { periodo dado; pago según unidades producidas o } \\
\text { espacio utilizado en las instalaciones de fabricación }\end{array}$ & Moderado-Alto \\
\hline Alianza estratégica & $\begin{array}{l}\text { Acuerdo a largo plazo; intercambio abierto de } \\
\text { procesos y propiedad intelectual; adaptabilidad; } \\
\text { comunicación recíproca frecuente }\end{array}$ & Alto \\
\hline
\end{tabular}

En segundo lugar, la fabricación por contrato permite a los OEM concentrarse en sus actividades más rentables, por ejemplo I\&D o ventas y marketing. Es cierto que IBM poseía el dinero y los conocimientos para invertir en la automatización de las fábricas, y la empresa estaba satisfecha de que su relación con Sanmina le permitiese igualar los precios de Dell. Pero los puntos de diferenciación de IBM son su excepcional ingeniería y servicios, ambas formas de especialización en las que pudo concentrarse gracias a la subcontratación.

En tercer lugar, las empresas pueden comunicarse y coordinarse entre sí con mayor eficiencia que nunca. En consecuencia, la lógica económica que alguna vez empujó a los OEM a desempeñar internamente casi todas las funciones especializadas ya no es aplicable. Internet está impulsando la mayoría de estas eficiencias, como también lo están haciendo los métodos estandarizados de producción, los procedimientos de gestión, los protocolos de comunicación electrónica y los formatos digitales de diseño promovidos por la Organización Internacional para la Estandarización, una federación de cuerpos nacionales de normalización. HP, por ejemplo, puede usar tecnologías tales como el intercambio electrónico de datos para transmitir especificaciones 
directamente desde sus departamentos de diseño a las máquinas y robots que se encuentran en la planta de un fabricante contratista. Medidas como éstas liberan a los OEM para separar sus actividades de innovación de sus actividades de producción.

En cuarto lugar, los sistemas de fabricación flexible permiten a los OEM reemplazar un producto por otro con poca antelación. Valmet Automotive, por ejemplo, fue capaz de comenzar a ensamblar el Porche Boxster sólo 7 meses después de cerrar el acuerdo de producción con el fabricante de automóviles. Ford produce 3 chasis diarios en sus fábricas, cada uno de los cuales puede acomodar 9 modelos diferentes de automóviles; esto permite a la empresa cambiar su producción rápidamente hacia los modelos que generan la mayor demanda de mercado.

Por último, la combinación de estandarización y fabricación flexible permite a los OEM reemplazar a los CM que muestran bajo desempeño o escasa cooperación con la misma facilidad con la que pueden reemplazar productos en declive. La naturaleza recíproca de estas relaciones -y, a la inversa, la capacidad de cada parte para retirarse a la primera señal de obstrucción por parte del socio- hace que sean fáciles de adoptar.

Los principales OEM no pueden darse el lujo de regresar a la seguridad de la integración vertical; los beneficios de la especialización son demasiado grandes. La mejor alternativa es dominar la etapa de desarrollo actual de la subcontratación, protegiéndose de las conductas oportunistas e interesadas de los fabricantes contratistas y de otros socios.

\section{CÓMO PUEDEN RESPONDER LOS OEM}

Claramente, los OEM no tienen más opción que coexistir con la fabricación por contrato. Afortunadamente, disponen de unas cuantas opciones defensivas para responder a sus peligros.

- Tenga cuidado con lo que subcontrata. Los procesos que son parte de las competencias centrales de un OEM o que involucran activos corporativos cruciales no deberían subcontratarse de ninguna manera. Sony Ericsson, por ejemplo, sólo subcontrata la fabricación de sus productos antiguos y que, por lo tanto, ya han sido copiados. Cisco Systems mantiene una capacidad interna de fabricación para sus routers y ordenadores de más avanzada tecnología y para sus prototipos. Aunque Alcatel comenzó 
en 2000 a vender la mayoría de sus cerca de 100 plantas -algunas de ellas a CM como Solectron y Sanmina-, retuvo media docena con el propósito de fabricar nuevos productos y artículos de alta tecnología que sólo pueden producirse en equipos de propiedad exclusiva.

En estos casos, el riesgo de infracción -el mayor de los costes de transacción- es alto. Y los volúmenes de ventas no serían tan grandes como para que la fabricación por contrato generase importantes economías de escala; sería demasiado pronto para que la tecnología se extendiera a los competidores, cuyos negocios adicionales los CM podrían luego traducir en menores costes unitarios para todos. Además, una empresa que ha subcontratado toda su fabricación perderá con el tiempo la mayor parte de sus conocimientos de fabricación, los cuales necesita, al menos, para supervisar e informar el trabajo de sus CM.

Esto explica que la incursión de Porsche en la fabricación por contrato no incluyera su serie 911 , que la empresa utiliza para introducir sus innovaciones. En su lugar, el contrato involucró al Boxster, un automóvil de lujo a ojos de muchos, pero que, sin embargo, es el modelo de entrada de Porsche. Si bien es cierto que Porsche recuperó en 2004 cerca de un tercio de la producción del Boxster, lo hizo en respuesta a la presión de los representantes sindicales en su consejo de administración por repatriar los puestos de trabajo que habían sido trasladados a Finlandia. Mientras tanto, la planta de Porsche en Leipzig, Alemania, continúa ensamblando los más nuevos y sofisticados modelos, Cayenne y Carrera GT.

Se podría esperar que los ejecutivos fueran conscientes del riesgo de externalizar sus competencias centrales. ¿Por qué están, entonces, tan ansiosos por subcontratar? La respuesta podría hallarse en varias influencias contrapuestas. La primera es la propensión de los ejecutivos a desprenderse de activos tangibles para aumentar el retorno sobre los activos de la empresa y su retorno sobre la inversión, y ser recompensados por ello. Además, al reducir el tamaño de la mano de obra, los ejecutivos normalmente pueden mejorar las tasas de productividad y evitar las largas y arduas negociaciones con los sindicatos; cambiar de proveedor es casi con certeza mucho más fácil.

- Adecue la relación a las circunstancias. Cuando el producto de un OEM no es novedoso ni único, su grado de innovación, complejidad y madurez en el mercado debería dictar la duración de su relación con el CM. Si la novedad y complejidad de un producto requieren que un CM 
destine tiempo y otros recursos a dominar su fabricación, necesitará el aliciente de un contrato a largo plazo para realizar esas inversiones. Un contrato a largo plazo también protegerá las inversiones propias del OEM tendentes a asegurar que el CM alcance la maestría del proceso de producción. En situaciones en que el producto del OEM sí es novedoso y complejo, se ha vuelto casi imposible para el OEM encontrar rápidamente un $\mathrm{CM}$ de reemplazo. Por consiguiente, un contrato a largo plazo se vuelve valioso porque dificulta que el CM abandone al OEM o exija condiciones prohibitivas como precio por su permanencia. Una consideración adicional para un OEM es que hará el CM con su propiedad intelectual cuando las dos partes ya no estén vinculadas legalmente. Los contratos que se redactan para anticipar y enfrentar estas eventualidades no pueden evitar ser complejos en sí mismos, aunque el coste y la dificultad de prepararlos se justifican debido a la seriedad de lo que está en juego.

A la inversa, si el OEM puede cambiar fácilmente de $C M$ porque el producto es simple de fabricar o ha existido durante el tiempo suficiente para volverse genérico, un contrato de menor duración sería práctico -incluso ventajoso- para el OEM. En esos casos, nada debería impedir que el OEM busque condiciones más atractivas de un CM diferente, o viceversa.

En 1999, DaimlerChrysler (en ese momento Daimler-Benz) pidió al fabricante contratista Magna Steyr que ensamblara su todoterreno Mercedes-Benz Clase M. La primera unidad salió de fábrica sólo 8 meses después del acuerdo inicial. En ese caso, un contrato de duración limitada fue todo lo que ambas partes necesitaron para proteger sus inversiones. Sin embargo, cuando BMW pidió a Magna que ensamblara su modelo X3, las partes prepararon y firmaron un acuerdo más largo. En este caso, BMW quería la ayuda de Magna para lograr adelantos en la tecnología de tracción $4 \times 4$, que proporcionaran al $X 3$ algo de la sensación de manejo por la que son conocidos los automóviles BMW.

Los acuerdos organizacionales entre OEM y CM abarcan desde contratos por una sola vez -conocidos como "acuerdos de mercado"hasta pactos más interdependientes y continuos, tales como acuerdos marco, joint ventures y otros tipos de sociedades. Un acuerdo de mercado, que podría o no ser renovable, involucraría la fabricación de, digamos, un tipo específico de reproductor MP3, y el pacto contendría detalles técnicos y de diseño muy precisos. En contraste, un acuerdo marco podría requerir que el CM produjese varios modelos de reproductores MP3 en un año determinado. Un acuerdo de sociedad, sin embargo, podría comprometer 
al CM como proveedor exclusivo y de largo plazo de los reproductores MP3 para un OEM.

Hay dos variedades interesantes de acuerdos a corto plazo. Elamex, un fabricante contratista de componentes electrónicos, deja que los clientes escojan entre un contrato "llave en mano" o un acuerdo de "cobertura". En el primero, un cliente de Elamex comparte la línea de montaje con otros clientes; cada uno le paga a Elamex según la cantidad de unidades que la empresa ha producido para ellos. En el segundo caso, se dedica una porción de la planta a la fabricación de los productos de un cliente específico, y éste paga una parte proporcional de los gastos generales, e incluso trae a sus propios ejecutivos para supervisar los procesos. Debido a que la empresa no posee capacidades de diseño, Elamex establece pocas alianzas a largo plazo. De aquí se desprende que la empresa prefiere trabajar con fabricantes experimentados antes que con firmas recién creadas.

Curiosamente, muchas alianzas estratégicas terminan retrocediendo hasta relaciones temporales de acuerdos de mercado. Esto ocurre debido a tres razones principales: primero, muchos OEM parecen perder de vista el propósito último de sus acuerdos a largo plazo y comienzan a presionar fuertemente a los CM para que produzcan ahorros. Como resultado, los $\mathrm{CM}$ comienzan a sentir que sus inversiones en aprender cómo fabricar un producto especializado o único no generarán un retorno. Quizás comprensiblemente, los CM sometidos a esta clase de presión abandonan cualquier escrúpulo que pudieran tener frente $a$, por ejemplo, vender directamente a los clientes del OEM. Esta es una situación especialmente peligrosa para el OEM cuando no puede encontrar fácilmente a otro CM capacitado, una de las principales razones para formar una sociedad a largo plazo en primer lugar.

Una segunda razón para la involución de las alianzas estratégicas es la inevitable devaluación de todo aquello que era nuevo y único acerca del producto. Este problema es particularmente pronunciado en el ámbito de la alta tecnología, donde los productos tienen ciclos de vida tan cortos. A medida que los productos se comoditizan, los OEM obtienen una amplia gama de proveedores intercambiables. Considere el caso de los PC: muchos fueron fabricados originalmente por los dueños de las marcas. Posteriormente, la tecnología de montaje de superficie, la creciente codificación del conocimiento y la sistematización de los procesos internos hicieron más fácil el ensamblaje de los PC y al alcance de las capacidades de proveedores externos, a quienes se subcontrató el trabajo. 
Hoy, la mayoría de los PC son productos genéricos compuestos de placas madre, ventiladores y unidades de disco duro adquiridos por un ensamblador local siguiendo las especificaciones de un OEM.

La tercera razón por la que se debilitan las alianzas estratégicas es la creciente modularización de componentes. La automatización demanda menos decisiones y menos improvisación de parte de los trabajadores. Y la ensambladura se ha vuelto más simple para los CM, ahora que los OEM están transfiriendo porciones cada vez más grandes de trabajos de fabricación desde los CM hacia sus proveedores, que son responsables de convertir partes en una única pieza de equipo, como por ejemplo el asiento de un automóvil. Ambos desarrollos hacen que sea menos importante para los CM poseer destrezas y conocimientos especiales.

- Otorgue libertad a sus socios de confianza. Como se mencionó antes, en situaciones de productos no comoditizados, los OEM deberían procurar relaciones cercanas con fabricantes contratistas de confianza para minimizar el riesgo de filtraciones de propiedad intelectual y proteger sus inversiones. Esta cercanía tendría el beneficio secundario de hacer que los CM se vuelvan dependientes de los OEM por financiación y orientación técnica. Pero estas relaciones no deberían ser exclusivas; de lo contrario, los OEM quedarán aislados de los innovadores del sector o se les negarán las máximas economías de escala posibles que los $C M$ pueden alcanzar al atender a muchos clientes. Los OEM que dependan de CM desvinculados de los desarrollos en el sector terminarán comercializando productos cuyo coste y calidad no son competitivos frente a los de su rivales.

Por consiguiente, un OEM debería establecer una relación cercana con un CM que ya haya tenido relaciones con otros OEM. El contrato debería permitir que los CM establezcan estas sociedades. De hecho, algunas empresas podrían esperar e, incluso, incentivar a sus CM para que encuentren otros clientes. En 1999, el fabricante contratista Flextronics compró una planta al OEM sueco Ericsson después de adjudicarse un contrato de esa empresa. Sólo un tercio de la capacidad de producción de esa fábrica está dedicada a productos de Ericsson; una porción mayor está dedicada a fabricar teléfonos inalámbricos, buscapersonas bidireccionales y otros dispositivos para Motorola. Al vender su fábrica a Flextronics, Ericsson ha fomentado una relación más estrecha entre Flextronics y uno de sus principales competidores, de la cual espera beneficiarse. También 
es improbable que esté descontenta porque Microsoft haya establecido un centro de investigación inalámbrica en Suecia, para estar cerca de este nodo de know-how de fabricación. Si Ericsson hubiese querido una relación exclusiva con su CM, seguramente habría encontrado un fabricante contratista dispuesto a aceptar sus condiciones, aunque probablemente habría sido un CM de segunda categoría.

En situaciones de productos no comoditizados, los OEM y los CM deberían comprometerse en relaciones a largo plazo, de manera que sus inversiones tengan tiempo suficiente para entregar retornos. Naturalmente, cuanto mayor es la duración de un contrato, más contingencias pueden producirse durante su transcurso y menos predecibles serán esas contingencias. Por consiguiente, tales contratos deberán ser altamente detallados, y los significados de las relaciones claramente explicados. En el caso de Magna Steyr y BMW, el montaje del X3 implicaba detalles tan complejos, técnicos y comerciales que el contrato tenía más de 5.000 páginas. Sin embargo, la aplicación incluso de un contrato como ése dependerá de la buena fe de las partes.

¿Cómo puede entonces un OEM fomentar la buena fe y el compromiso de un $\mathrm{CM}$ con la alianza cuando el CM tiene también otros jefes? Un OEM podría comenzar tratando de adquirir una percepción de las posibilidades de confianza a largo plazo. La mejor forma de hacer esto es examinar el comportamiento anterior de un CM; eso podría implicar hablar con otros OEM, con representantes de asociaciones gremiales y con los proveedores del CM. Un OEM también puede investigar la historia de disputas comerciales del CM. Si un CM intenta persuadirlo de que contrate sus servicios ofreciéndole compartir los secretos comerciales o la propiedad intelectual de otros clientes, es bastante probable que más adelante haga lo mismo con su propiedad intelectual.

Aunque se hayan establecido las buenas intenciones de un potencial CM, un OEM deber determinar su fiabilidad. Antes de contratar a un proveedor, por ejemplo, Toyota y Honda examinan detenidamente su proceso de producción y su estructura de costes. Tras seleccionar a un proveedor, los fabricantes le envían un cuestionario mensual solicitando una historia de desempeño: calidad y cantidad de su producción, rapidez en la entrega, ocurrencia de hechos inusuales. Los ingenieros de Toyota y Honda pueden luego pasar varios meses en las instalaciones del proveedor, usando la información obtenida para resolver problemas y mejorar los procesos. 
Por último, un OEM necesita desarrollar una buena comunicación. Esto implica algo más que realizar vigilancia y reaccionar a las respuestas que ésta genere. Ambas partes necesitan compartir sus objetivos para la alianza y convenir normas, valores y procedimientos. El intercambio de personal ayuda a facilitar esas cosas. Los OEM no sólo pueden enviar a sus ingenieros a las instalaciones del CM, sino que también pueden crear, como ya han hecho Toyota y Honda, programas de "ingenieros visitantes" mediante los cuales los proveedores de primer nivel envían a varios de sus ingenieros de diseño a las oficinas del OEM entre dos y tres años.

Un OEM para el que la viabilidad comercial de su producto descansa en tecnología y procesos patentados, necesita que su CM conozca bien el negocio. El OEM debe tomar medidas, tanto al principio de la sociedad como durante su transcurso, para asegurarse de las competencias del CM, la actualidad de sus conocimientos y sus buenas intenciones.

- Combata la deslealtad del CM profundizando la lealtad de sus distribuidores y clientes. Puede ser que los CM tengan ciertas ventajas, siendo las más importantes su superior destreza de fabricación, una mayor variedad de productos que ofrecer y costes más bajos. Pero el auge de la fabricación por contrato también ha traído aparejados algunos desafíos para los CM que deciden vender los productos que fabrican. Entre ellos está la proliferación de opciones y de marcas para el consumidor que ha sido estimulada con la llegada de la fabricación por contrato. Hoy en día, existen, por ejemplo, alrededor de 20 marcas globales de televisores. Aunque los CM por definición han superado las barreras para la introducción al negocio de la fabricación, las marcas bien establecidas de los OEM y su experiencia en marketing y ventas constituyen otro tipo de barreras. Además, los OEM pueden profundizar sus actuales relaciones directas con clientes y distribuidores más fácilmente de lo que les costara a los CM crear esas relaciones desde cero.

Los OEM pueden fortalecer sus lazos con los clientes, ofreciendo recompensas o descuentos especiales a aquellos que compran frecuentemente sus productos, mediante la personalización de sus relaciones con clientes y el ofrecimiento de un sólido soporte técnico. Algunos OEM publicitan productos profesionales directamente a los usuarios finales, con la esperanza de que dichos usuarios expresen una preferencia a los compradores reales. Philips Medical Systems, por ejemplo, ha lanzado una 
campaña masiva por TV en varios países para publicitar sus escáneres Brilliance $\mathrm{CT}$, los cuales se venden exclusivamente a los hospitales.

Sin embargo, la lealtad de los clientes debe descansar fundamentalmente en lo que distinga al producto de un OEM de los productos de la competencia. La lealtad no puede estar basada en precios bajos, ya que aquellos consumidores que busquen el mejor precio correrán el perpetuo riesgo de cambiarse, y los $C M$ estarán casi siempre en mejor posición que los OEM para ofrecer el mejor precio. Lo mismo es aplicable a la calidad, que queda asegurada por la excelencia operacional del CM, y a la gama de productos, que es una característica de la escala del CM. De hecho, los productos que los $\mathrm{CM}$ fabrican para otras marcas serán similares en calidad y coste, precisamente porque han sido fabricados por la misma empresa. En contraste, un $\mathrm{CM}$ que fabrica productos únicos para un OEM determinado estaría atado por un acuerdo a largo plazo que lo eliminaría como una amenaza competitiva.

En TI, los ensambladores de "clones" no sólo ofrecen los mejores precios, sino también mayores posibilidades de personalización que las empresas que venden productos diferenciados, pero fijos. Los clientes de los ensambladores escogen componentes de distinta calidad y así obtienen ordenadores hechos a la medida en sus niveles deseados de precio. Es probable que los automóviles se ensamblen justamente de esta manera en un futuro no muy lejano; la fabricación flexible y la cantidad siempre decreciente de tiempo y dinero que cuesta transportar un automóvil por vía marítima (tres semanas y $500 \$$ entre dos puntos cualquiera del mundo) pronostican el surgimiento de automóviles de marca de los propios CM en países de bajos salarios como China.

En consecuencia, las empresas que deseen adoptar una estrategia de diferenciación tendrán que enfocarse en la investigación, el diseño, las ventas, el tiempo de llegada al mercado o el servicio al cliente. Al hacerlo, se estarán comprometiendo a una subcontratación amplia. Dell, desde luego, ha resuelto el problema de la comoditización del hardware eliminando intermediarios -lo que le ha permitido acercarse a sus clientes- y ofreciendo mejor servicio y soporte.

Los OEM también pueden tratar de reconstruir la lealtad de los distribuidores. Éstos pueden ser inusualmente receptivos a las propuestas de los CM, porque resienten el hecho de verse presionados por los OEM que buscan ahorros a corto plazo. Incluso en aquellos casos en que los OEM actúan generosamente, los temores de desintermediación de los 
distribuidores y su reemplazo final por canales electrónicos, los tientan a aceptar a cualquier empresa que prometa un trato justo y relaciones de larga duración. Los OEM que se comprometan de forma creíble con los distribuidores, aunque éstos puedan implicar costes más altos, pueden contribuir a vincular los distribuidores con las marcas de los OEM y mantener a raya a los CM más agresivos. Estos acuerdos pueden incluir pagos para entrenar a personal del distribuidor en el servicio y reparación de productos de OEM; financiar el coste de un sistema de procesamiento que responda a los requerimientos de ambas partes, o la incorporación de la identidad del distribuidor a las campañas de marketing del OEM. Esta última, en particular, debería disuadir al distribuidor de pensar en transformarse él mismo en una marca. Los OEM podrían también considerar otorgarle exclusividad territorial a un distribuidor, lo que haría mejorar los ingresos de este último e incentivarlo a especializarse en los productos del OEM. Si el distribuidor de todas formas estuviese tentado a confiar su lealtad a un nuevo OEM, sabe que tendría que pagar el coste de aprender la forma de vender y prestar servicio a la nueva marca.

- Mire más allá de los peligros de su propio mercado. Los desafíos estratégicos presentados por la fabricación por contrato hacen dudar sobre la obviedad de que la especialización triunfa sobre la diversificación. Tradicionalmente, las grandes empresas han mantenido carteras de patentes que son mucho más diversas que sus carteras de actividades de producción. En otras palabras, un buen OEM probablemente tendrá propiedad intelectual relacionada con productos distintos a sus productos principales. Un fabricante de puertas de automóviles, por ejemplo, necesita tener conocimientos acerca de los plásticos, bolsas de aire, sistemas electrónicos y cristales que forman parte de dichas puertas y podría incluso tener patentes de dispositivos que ha inventado en el transcurso de la investigación y desarrollo de mejoras de su tecnología principal. Los OEM podrían considerar explotar ese conocimiento adicional o secundario, introduciéndose en nuevos mercados. Ese tipo de entrada podría ser facilitada por los CM contratados por los OEM, de la misma manera que los competidores usan la fabricación por contrato para introducirse en los mercados originales de los OEM. En este caso, los OEM estarían sacando ventaja del mismo recurso usado para atormentarlos. Los OEM lograrían esto haciendo a los competidores de algún otro mercado lo mismo que les habían hecho antes a ellos; a bajo coste y bajo riesgo, gracias a las características de la fabricación por contrato. Estos nuevos productos descansarían, lo cual es una ventaja, en tecnologías nuevas y 
patentadas. Por ejemplo, Royal Philips, una empresa electrónica, ya diseña y vende una amplia gama de productos: ordenadores, equipos de fotografía, reproductores portátiles de discos compactos, frigoríficos. De manera similar, las empresas de automóviles tendrían la credibilidad de marca y las capacidades tecnológicas para diversificarse hacia los mercados de consumible, aleaciones, baterías, filtros, espejos, recubrimiento de vidrio, motores eléctricos y dispositivos y sistemas de seguridad. Al disponer de $C M$, los cuales esperan ansiosamente entrar en acción, lo único que esas empresas y otras requerirían para comenzar es suficiente experiencia en producción para ensamblar prototipos y productos de alta calidad en cantidades limitadas. Toyota se ha diversificado hacia las telecomunicaciones, casas prefabricadas y embarcaciones deportivas, lo que hizo de forma oportunista, no porque algún CM haya puesto presión a los márgenes de la empresa.

Los OEM deberían implementar esta estrategia para introducirse en mercados distintos, pero relacionados con sus productos centrales, en los que sus marcas puedan tener alguna influencia. Para los OEM, la explotación directa de sus patentes -con la ayuda de sus CM- es una alternativa mejor para lidiar con una acumulación de propiedad intelectual no utilizada, de lo que podría ser el licenciamiento externo. Sin la fabricación por contrato, la mayoría de los OEM probablemente nunca intentaría una entrada directa al mercado.

Mientras la innovación tecnológica continúe aumentando los beneficios de la especialización, la fabricación por contrato será una opción ejercida a menudo por los OEM. Lamentablemente, los incentivos de los ejecutivos fomentan un enfoque generalista y poco crítico frente a las decisiones de cuánto subcontratar, qué productos subcontratar, a qué CM contratar y bajo qué forma: un acuerdo de mercado, una alianza estratégica o una fórmula intermedia. Cuando los OEM comparten su propiedad intelectual con los $C M$, es importante que la relación sea cercana y de confianza, pero no tan cercana como para que los CM pierdan contacto con el mercado y con las contribuciones de otros OEM. Los OEM deberían tratar a sus clientes y distribuidores lo bastante bien como para que estos últimos se vuelvan inmunes a las atracciones de los CM, y deberían disminuir el riesgo diversificando su cartera de productos. 